\title{
EDITORIAL
}

\section{Animal models for evaluating psychotropic drugs}

There is still no sure way of determining in animals any elements, precursors, or equivalents of human intrapsychic events and hence much of the pertinent evidence from studies of animal behaviour is at best circumstantial. In the context of 'aberrance' such information is doubly unsatisfactory since the aetiology of most psychiatric conditions is obscure and very little is known about naturally occurring behaviour disorders in animals. In spite of these uneasy foundations, the demands upon workers in this broad field of research continue to increase - the preclinical evaluation of psychotropic drugs is a notable example. The experimenter can find himself trying to identify or reproduce features of human syndromes in animals, but without the necessary basic information and without adequate means of checking the validity of his assumptions. On the one hand, he risks being admonished for trying to mimic epiphenomena, and, on the other, for oversimplifying his constructs to such a degree that applications to man are highly questionable. It is not surprising, therefore, that extrapolations from animal experimental data to the psychiatric clinic are closely hedged with caveats.

The basic issues involved have not changed over the years and the central problem has always been that of determining homology - that is, are the behaviour patterns being compared in different species alike both in origin and fundamental structure? It seemed sensible to adopt this criterion from comparative anatomy, but the position has not changed greatly since Russell and his colleagues (National Institute of Mental Health, 1962) noted . . ' at the present stage of the development of the behavioural sciences it is difficult to establish that apparently similar behavioural patterns are truly homologous'. A broad strategy for research was therefore recommended-in particular, for evaluating psychotropic drugs in animals, but it is of general interest.

It contained a series of steps: (1) the analysis of a human aberrant behaviour pattern for its basic characteristics; (2) the selection of aspects of animal behaviour as similar as possible to these characteristics; (3) a search for chemical agents which might affect these characteristics in animals; (4) tests of the effects of these agents on the human behaviour patterns originally analysed.

These guidelines appear worthwhile and practical but they are not generally followed in the pharmaceutical industry, where the emphasis is on comparisons of empirically derived profiles of new substances with those of established drugs. The shortcomings of this latter strategy, sometimes described as a criterion drug approach, are well known, the most important being the production of 'me-too' relatives at the expense of excluding drugs with novel and unexpected actions. The output of 'new' drugs in the past few years, with one or two exceptions, amply justifies these criticisms.

The reasons behind adopting the criterion drug-as opposed to the criterion behaviour-approach which was embodied in the proposals of Russell and his colleagues (National Institute of Mental Health, 1962) must in part reflect commercial pressures but an important pragmatic factor may be that the behaviourally oriented method is inherently unworkable. The search for behavioural homology, or approximations to it, was implicit in the first two steps of the strategy outlined above. In order to be meaningful, the process of selection of basic characteristics from a given human syndrome can hardly ignore questions of origin and of fundamental structure. Knowledge of these matters would make recourse to animal models of academic interest and its lack underlines the fallacy of trying to seek homologies along unspecified parameters. The only way left, then, is to construct parallels based upon associated physiological and gross behavioural symptoms, with the theoretical bias of the experimenter determining the degree to which a priori assumptions are made about underlying unitary lesions or disease processes. With these limitations in mind, it is hardly surprising that nearly all the major therapeutic discoveries have been "in large measure due to chance, 
the prepared mind and serendipity' (Lasagna, 1964). Occasionally, when drugs have been introduced on purportedly rational grounds they have made little therapeutic impact, and the prospect of having to wait for future inspired clinical observations in unexpected settings is a gloomy one. It is also possible that, as in the past, incorrect hypotheses will sometimes lead to major advances; the logic behind the very first attempts to introduce into psychiatry treatments such as ECT, lithium, and the benzodiazepines can be criticized, while in no way diminishing the importance of the discoveries themselves.

Progress in psychopharmacology has been likened to a process of successive approximations (Steinberg, 1964) in which the two main research tools, drugs and behaviour, are progressively refined with varying emphasis placed upon them in differing studies. The problem, as always, lies in interpreting the data and a natural trend has been to seek for some system and coherence in the increasingly credible neurochemical and physiological substrates of behaviour. The most strikingly successful example of this is provided by the introduction of L-dopa for the treatment of Parkinson's disease. The induction of 'turning' behaviour in rats by lesions in, and/or chemical and electrical stimulation of, the nigrostriatal pathways has provided a most valuable technique (Ungerstedt, $1971 \mathrm{a}, \mathrm{b}$ ). Nevertheless, turning behaviour is not a model of Parkinson's disease; it is a test in rats for studying the effects of specific neuronal disturbances of dopamine. Successful extrapolations to man have depended upon the results of research into the distribution and levels of dopamine and its metabolites in normal and affected human subjects.

\section{MODELS OF SOME PSYCHIATRIC DISORDERS}

The elusiveness of structural and other lesions in psychiatric conditions such as schizophrenia and affective disorders has handicapped attempts to develop rationally derived treatments for these states. There have, however, been some recent interesting and provocative developments which illustrate very well the present state of the art of extrapolating between species.

\section{SCHIZOPHRENIA}

There are several clues which suggest that disorders of dopamine may underlie aspects of schizophrenic illnesses. The evidence is derived from the possible relationship between the therapeutic potency of neuroleptic drugs and the induction of extrapyramidal effects through some common central action such as blockade of dopamine receptors (Van Rossum, 1966; Randrup and Munkvad, 1972; however, for contrary evidence see Crow and Gillbe, 1973, 1974). Animal studies suggesting that amphetamine-induced stereotyped behaviour is mediated by dopamine (Randrup and Munkvad, 1972) are linked with observations of similar patterns of behaviour in amphetamine psychosis. Similarities between the relative potencies of the stereoisomers of amphetamine in producing stereotypy in animals (Snyder, 1972) and psychosis in human subjects have led Angrist et al. (1971) to suggest that 'animal stereotypy ... should be utilized as an animal model for the human stimulant psychoses', and to point to 'the striking clinical similarity of amphetamine psychosis to paranoid schizophrenia'. The observer is left to ponder the question of common underlying disturbances (Snyder, 1973) in these two conditions which are by no means identical.

Carlsson et al. (1973) have reported that concurrent treatment with the tyrosine hydroxylase inhibitor, $\alpha$-methyl-p-tryosine, potentiates the antipsychotic effects of phenothiazine and butyrophenone drugs. They suggest that, when neuroleptic drugs block central catecholamine receptors, this then induces a compensatory increase in the presynaptic synthesis and release of these amines. Partially inhibiting catecholamine synthesis with $\alpha$-methyl-p-tyrosine might overcome this putative feedback mechanism and thus enhance the therapeutic effects of these drugs. Both the clinical observations and the suggested underlying mechanisms need further evaluation, but this work illustrates how, at one level, a neurochemical hypothesis can be studied in intact animals without recourse to approximations of the human pathological state. In a behavioural test of the interactions between the 
two treatments, one sensitive measure was found to be operant responding for food by rats (Ahlenius and Engel, 1971).

Another model for schizophrenia has been proposed (Stein and Wise, 1971) in which it is suggested that an endogenous (abnormal) accumulation of 6-hydroxydopamine results in the deterioration of noradrenergic pathways that mediate reward in the brain; the experiments were done in rats responding for electrical stimulation of such pathways. There is, however, some difficulty in reconciling this intriguing hypothesis with the clinical complexities of schizophrenic states; the authors select as two primary symptoms of schizophrenia the deficit in goal-directed thinking and the capacity to experience pleasure, both of which are assumed to reflect impairment of noradrenergic reinforcement pathways. Detailed studies of the ways in which both dopaminergic and noradrenergic fibres can sustain electrical self-stimulation behaviour (Crow, 1972; 1973) and also of interactions between different fibre systems do not provide unqualified support for formulations in terms of unitary neurophysiological 'lesions', in this particular case resulting in 'a regression to primitive and less goaldirected modes of behaviour regulation' (Stein et al., 1972).

\section{MANIC-DEPRESSIVE DISORDERS}

Recently, Ashcroft and his colleagues (M.R.C. Brain Metabolism Unit, 1972) have presented a modified amine hypothesis for the aetiology of affective illness. They emphasize attempts to differentiate sub-groups of patients on both behavioural and metabolic measures and then to link these with observations of the behaviour of animals treated in such ways as to affect the functioning of central transmitter systems. These authors make the point that 'the feeling state and cognitive aspects can only be examined in man . . . It seems however that certain of the behavioural and autonomic changes may have much in common in man and other species'. The methods for inducing such changes in animals do pose a problem, for if surgical or chemical lesions are required to demonstrate these concomitants of 'mood' then their validity is somewhat suspect. Ashcroft and his colleagues (M.R.C. Brain Metabolism Unit, 1972) were obliged to refer to anatomical and functional similarities of higher mammalian nervous systems, disturbances of which result in fragments of behaviour 'which may be the building blocks of more complex adaptive reactions'. A parallel is drawn between chemically induced stereotypies in animals and in man, and apparently purposeless, repetitive behaviour patterns that are sometimes seen clinically-for example, in hypomanic patients or in agitated depression. If such a crucial emphasis is to be placed upon changes in exploratory and stereotyped behaviour patterns in depression and in hypomania, rather careful clinical evaluations will be needed of endogenous and chemically induced patterns of stereotypy in man. Would the occurrence and degree of these types of behaviour in the premorbid state have any predictive validity? In animal tests-for example, in rats-the possible value of measures of exploration and stereotypy can lead one to overlook how relatively unsubtle they may be as indices of central transmitter events (Norton, 1973). Measures of apparent purposive locomotor activity, sometimes in novel situations, are generally taken to reflect exploration but there is evidence that amphetamine in low doses actually reduces investigatory behaviour while increasing ambulation. At higher doses stereotypy occurs, but, since purposive locomotion and stereotypy are mutually exclusive components of a rat's repertory of expressed behaviour, this must complicate inferences about dynamic changes in transmitter systems.

\section{ANXIETY STATES AND BEHAVIOUR MODIFICATION}

The history of systematic attempts to create animal models of human neurosis dates from th. celebrated studies in dogs in Pavlov's laboratory and many reasons have been advanced why these and subsequent experiments have failed to improve substantially our understanding of human neurotic disorders. A prominent feature of such experiments has been the artificiality (for the animal) of the test situation. Nowadays this can be exceedingly complex : lights wink, buzzers sound, electric shocks may be delivered and can be delayed if the animal presses a lever which sometimes also produces food or water. This can result in an exceptional tendency in the subject to suppress its normal 
responses to such happenings, and in the experimenter, to record only what he set out to measure. Thus when Davis (1968) wrote,

'although it is tempting to discuss the effects of reserpine on "fear" or on "anxiety", it is more productive to discuss the effects of reserpine (i.p.) at $1 \mathrm{mg} / \mathrm{kg}$ on conditioned suppression, or more accurately, on conditioned suppression of bar pressing in male Sprague-Dawley rats under a variable interval schedule $(\bar{X}=30 \mathrm{sec}$, range $=5-90 \mathrm{sec}$ ) of $45 \mathrm{mg}$ dry food reinforcement, during the presentation of a $1250 \mathrm{cps}$ tone for $60 \mathrm{sec}$ at $92 \mathrm{db}$ upon which the grid delivery of a $2-\mathrm{sec} \cdot 8 \mathrm{~mA}$ electrical shock was contingent',

he diminished one source of ambiguity but screwed on the lid of the black box even more firmly. There is no denying the place of such experiments in furthering our understanding of how animals learn, for example, to avoid traumatic events such as shocks and to respond to cues which signal food, but questions do arise about the general value of the information so obtained, even within the species and strain being studied. To take one example, signalled electric shocks are widely used to influence animal behaviour, but it is permissible to ask (Bolles, 1970) whether owls normally hoot a few seconds before pouncing on a rat. Similarly, a rat's behaviour is very different when it has to learn to run and jump to avoid shocks than when it has to press a lever. Psychiatrists and psychologists have begun to look carefully at concepts such as 'preparedness' (Marks, 1969; Seligman, 1970) and at species-specific patterns of responding (Bolles, 1970); these radically challenge the premises of general learning theories which hold that all events are equally associable.

In contrast with the immense literature on avoidance of electric shocks by animals there seems only recently to have been an interest in avoidance of other sorts of aversive stimuli. Following on the studies of Garcia and his colleagues (see reviews by Revusky and Garcia, 1970; Seligman, 1970; Rozin and Kalat, 1971), it has become clear that animals can learn to avoid taste cues often after a single pairing with an internal aversive state-for example, nausea after poisoning or irradiationand that such associations can be formed with great facility. This is in marked contrast with attempts to demonstrate avoidance of noises or lights which have been repeatedly paired with nausea, or tastes paired with electric shocks; there appears, therefore, to be some specificity in this regard. In the light of such observations, there has been a perceptible shift among psychologists towards the recognition of some sort of continuum between instinctive and acquired behaviour. There are also other findings which may be of interest to psychiatrists: the avoidance of taste cues for poisons can develop even if there is a delay of several hours between the presentation of the conditioned stimulus and the onset of the internal, aversive, unconditioned stimulus. It seems unlikely that intermediate cues, such as aftertastes, function as mediators for such learning. One can speculate whether analogous processes may not be involved in the genesis of phobic behaviour. For example, are there particular types of aversive states which link readily with certain conditioned stimuli and not with others; would direct contiguity be unnecessary for the subsequent development of avoidance responses? If some specificity of conditioned and unconditioned stimuli could be identified in this rapid learning paradigm, would not similar considerations be relevant to the modification of such behaviour once it had been acquired?

Techniques of aversion therapy frequently employ discrete episodes of externally applied punishment which are made contiguous with, or consequent on, the behaviour to be changed (Rachman and Teasdale, 1969). Their limited value may partly derive from the fact that behaviour which is acquired in the rather special way discussed above, is less likely to be affected than behaviour that develops gradually over repeated trials. Furthermore, the aversive states engendered by experimentally contrived episodic punishment-for example, electric shocks-may not be biologically relevant to all kinds of maladaptive behaviour patterns and attempts to treat overeating by pairing favourite foods with highly unpleasant odours (Foreyt and Kennedy, 1971) are especially interesting in this context. There are difficulties, however, even when such therapeutic models are made more specific. For example, one might predict that aversion treatment of alcoholism with disulfiram would be more potent than aversion with electric shocks. Yet one must acknowledge that in this case severe nausea and sickness are relatively poor at inducing 'poison' avoidance of alcohol in physically dependent 
heavy drinkers. But it should be possible to test whether abstinent, hospitalized alcoholics given controlled exposures to alcohol and disulfiram would subsequently show a diminished tendeny to relapse.

The efficacy of response prevention in modifying human and animal avoidance behaviour is partly due to the fact that prolongation of the conditioned stimulus renders it ineffective as a cue (Katzev, 1967). However, relatively few trials are needed and an equally important aspect of these procedures may be that the intensely unpleasant emotional state which arises during 'flooding' serves to punish and hence inhibit the subsequent expression of avoidance responses. Critical features of the aversive state must be its intensity and duration and also, especially, its qualitative relevance. This formulation, which focuses primarily on behaviour, is necessarily restricted in its scope and it ignores, for example, questions of meaning, gain, and manipulation.

In animal studies shocks are almost exclusively used as unconditioned stimuli in experiments on the conditioned suppression of appetitive behaviour. It may be that other types of aversive stimuli cannot easily be paired with neutral cues in order reliably to induce conditioned emotional reactions. More detailed explorations of such questions (for example, Best et al., 1973) must increase the validity of animal indices of conditioned fear while perhaps restricting their range. There is one essential proviso: the animal tests cannot be expected to produce plausible models of anxiety states while so little is known in man about relationships between 'normal' fear and morbid anxiety. If extrapolations from animals are to have any substance at all in this context, it must at least be demonstrated that a number of 'known' drugs for treating morbid anxiety have consistent effects on analogous tests of experimentally contrived fear in both animals and in man. Drugs such as the benzodiazepines and barbiturates have palliative effects in anxiety states and withholding these drugs typically results in a return of symptoms. Experimental observations in animals (Kumar, 1971a,b) are consistent with clinical findings. This type of drug-induced 'symptom relief' is sometimes confused with the rare phenomenon of state-dependent learning, which is also a function of the presence or absence of a drug in the system. The critical difference is that a drug must first be shown to modify learning or extinction before there is any question of assessing whether the persistence of such newly acquired changes depends upon the maintenance of the drug state of the subject.

\section{DRUG DEPENDENCE}

There is one field of research in which there has been surprisingly good agreement between human and animal findings: this is the self-administration of drugs and the development of behavioural and physiological dependence. The great majority of the relevant animal studies have been done in rodents and in primates. Screening methods in animals for drugs with dependence-inducing properties now play an important part in drug evaluation programmes. Data from biochemical and physiological research therefore can be linked with behavioural observations in intact animals and, allowing for interspecific variations, such work provides a coherent framework for clinical investigations of addiction. The very considerable value of such laboratory data might lead one to overlook an apparent paradox-anecdotal reports of drug-seeking behaviour by unconstrained animals in their natural habitats (Forsyth, 1968; Siegel, 1973) do not adequately counter the impression that the repeated voluntary ingestion of centrally acting substances is by and large a peculiarly human pastime.

\section{SOME GENERAL CONSIDERATIONS}

Advances in pharmacokinetics have done much to increase the predictive value of tests of drugbehaviour interactions in animals. The discussion here has been around a perhaps more fundamental source of interspecific variation. In the context of drug studies this is described as pharmacodynamic variation, and it is due to inherent differences between the organization and function of integrated systems in intact animals, including limitations of their capabilities. As a practical illustra- 
tion, studies of antiemetic drugs in rats and mice would be unproductive, since these animals cannot vomit. In the examples of recent research discussed earlier, it was tacitly assumed that, since behavioural and physiological indices covaried with disturbances of feeling, thinking, and perceiving in man, analogous indices in animals could serve as useful tools provided that certain criteria were met. A number of interesting speculations automatically arise, the two poles of which are either that human mental disturbances are more complex but nevertheless homologous versions of more primitive (unknown) mammalian disorders or, alternatively, that they are a new penalty, in an evolutionary sense, for having higher mental development-for example, the ability to think and talk, of itself generates the possibility of disorders of these faculties. This statement could equally well be made in terms of novel central transmitter system interactions and it incorporates the possibility of either reactive or endogenous disorganization.

The underlying issues are sometimes confused, although the implications for research are crucially important. There is no validity to the concept of a phylogenetic continuum with man at the top of a mammalian hierarchy, yet the very process of searching for animal models of human disorders induces a tendency to anthropomorphize and vice versa. A naked ape may more easily ignore Scott's (1967) warning not to think of monkeys as small people with fur coats. The search for valid animal models and for structural and functional 'common denominators' must therefore take into account the divergent lines of the phylogenetic tree. In the light of this it seems wishful to expect, for example, that analogous behavioural studies in a variety of species will necessarily illuminate subtle motivational changes that may be induced by centrally acting drugs. Apart from purely empirical evaluations, it is difficult to see how batteries of tests of operant responding applied to monkeys, rats, and pigeons can be meaningfully interpreted. Indeed, the outcome tends to be an emphasis on the reinforcement schedules employed with, consequently, a diminished appreciation of species differences and independent lines of development. Attempts to take account of such factors are hindered, as Hodos and Campbell (1969) have pointed out, by the fact that evolutionary insights cannot be derived in the usual way, since there are no behavioural fossils.

In spite of, or perhaps because of, all these hazards, the comparative approach to behaviour is both challenging and exciting and it allows little room for fixed opinions and expectations. There is no a priori reason why even if homologous patterris of normal or aberrant behaviour are adequately characterized, they should necessarily resemble each other any more than a bat's wing does a hand. Alternatively, through ecological convergence, analogous forms may in fact represent the end points of quite different lines of development. Thus it is extremely difficult to interpret the few recorded instances of self-mutilative behaviour in animals (Lester, 1972); the apparent resemblance between 'superstitious' behaviour in animals and obsessive-compulsive behaviour in man poses similar problems. In the same vein, Roth and Kerr (1970) have commented that both depression and suicide are unknown quantities in animals, and this could apply to any psychiatric disorder. The definition of a behavioural event such as suicide in man is of little help; how would one know whether an animal had committed suicide?

The main need, in the first instance, seems to be for a closer dialogue between the clinic and the laboratory and recent developments in formulating and studying behavioural analogies of depressive disorders are good examples of this. Studies of learned helplessness (Seligman et al., 1971), of disturbances of dominance hierarchies (Price, 1967), or of lasting consequences of mother-infant separations (Hinde and Spencer Booth, 1971) all attempts to tackle different aspects of the problem under controlled conditions in laboratory animals. The extent to which the findings are meaningful in terms of man can be established only by further research to which such studies themselves serve as pointers. The data cannot, however, provide more than a basis for speculation until matching clinical information has been gathered in man to check the validity of the assumptions being tested; this is a recurring need throughout the whole field. A telling point was made by Tinbergen (1968) in a slightly different context: 'Psychiatrists, at least many of them, show a disturbing tendency to apply the results rather than the methods of ethology to man'. 


\section{REFERENCES}

Ahlenius, S., and Engel, J. (1971). Behavioral effects of haloperidol after tryosine hydroxylase inhibition. European Journal of Pharmacology, 15, 187-192,

Angrist, B. M., Shopsin, B., and Gershon, S. (1971). Comparative psychotomimetic effects of stereoisomers of amphetamine. Nature, 234, 152-153.

Best, P. J., Best, M. R., and Mickley, G. A. (1973). Conditioned aversion to distinct environmental stimuli resulting from gastrointestinal distress. Journal of Comparafive and Physiological Psychology, 85, 250-257.

Bolles, R. C. (1970). Species-specific defense reactions and avoidance learning. Psychological Review, 77, 32-48.

Carlsson, A., Roos, B.-E., Wálinder, J., and Skott, A. (1973). Further studies on the mechanism of antipsychotic action: potentiation by $\alpha$-methyltyrosine of Thiordazine effects in chronic schizophrenics. Journal of Neural Transmission, 34, 125-132.

Crow, T. J. (1972). Catecholamine-containing neurones and electrical self-stimulation: 1. a review of some data. Psychological Medicine, 2, 414-421.

Crow, T. J. (1973). Catecholamine-containing neurones and electrical self-stimulation: 2. a theoretical interpretation and some psychiatric implications. Psychological Medicine, 3, 66-73.

Crow, T. J., and Gillbe, C. (1973). Dopamine antagonism and antischizophrenic potency of neuroleptic drugs. Nature, 245, 27-28.

Crow, T. J., and Gillbe, C. (1974). Brain dopamine and behaviour: a critical analysis of the relationship between dopamine antagonism and the therapeutic efficacy of neuroleptic drugs. Journal of Psychiatric Research. (In press.)

Davis, H. (1968). Conditioned suppression: a survey of the literature. Psychonomic Monograph, Suppl. 2, 283-291.

Foreyt, J. P., and Kennedy, W. A. (1971). Treatment of overweight by aversion therapy. Behaviour Research and Therapy, 9, 29-34.

Forsyth, A. A. (1968). British Poisonous Plants. 2nd edn. Bulletin No. 161. Ministry of Agriculture, Fisheries and Food. H.M.S.O.: London.

Hinde, R. A., and Spencer-Booth, Y. (1971). Effects of brief separation from mother on rhesus monkeys. Science, 173, 111-118.

Hodos, W., and Campbell, C. B. G. (1969). Scala naturae: why there is no theory in comparative psychology. Psychological Review, 76, 337-350.

Katzev, R. (1967). Extinguishing avoidance responses as a function of delayed warning signal termination. Journal of Experimental Psychology, 75, 339-344.

Kumar, R. (1971a). Extinction of fear. 1. Effects of amylobarbitone and dexamphetamine given separately and in combination on fear and exploratory behaviour in rats. Psychopharmacologia, 19, 163-187.

Kumar, R. (1971b). Extinction of fear. 2. Effects of chlordiazepoxide and chlorpromazine on fear and exploratory behaviour in rats. Psychopharmacologia, 19, 297-312.

Lasagna, L. (1964). On evaluating drug therapy: the nature of the evidence. In Drugs in our Society, Edited by P. Talalay. Johns Hopkins Press: Baltimore.

Lester, D. (1972). Self-mutilating behavior. Psychological Bulletin, 78, 119-128.

Marks, 1. M. (1969). Fears and Phobias. Heinemann: London.

Medical Research Council Brain Metabolism Unit (1972). Modified amine hypothesis for the aetiology of affective illness. Lancet, 2, 573-577.

National Institute of Mental Health (1962). Behavioral Research in Preclinical Psychopharmacology: Issues of Design and Techniques. Prepared by The Pharmacology Unit, and The Committee on Preclinical Psychopharmacology,
Psychopharmacology Service Center. U.S. Public Health Service Publication No. 968. Government Printing Office: Washington.

Norton, S. (1973). Amphetamine as a model for hyperactivity in the rat. Physiology and Behavior, 11, 181-186.

Price, J. (1967). The dominance hierarchy and the evolution of mental illness. Lancet, 2, 243-246.

Rachman, S., and Teasdale, J. (1969). Aversion Therapy and Behaviour Disorders. An Analysis. Routledge: London.

Randrup, A., and Munkvad, I. (1970). Biochemical, anatomical and physiological investigations of stereotyped behaviour induced by amphetamines. In Symposium on Amphetamine and Related Drugs, pp. 695-713. Edited by E. Costa and S. Garattini. Raven Press: New York.

Randrup, A., and Munkvad, I. (1972). Evidence indicating an association between schizophrenia and dopaminenergic hyperactivity in the brain. Orthomolecular Psychiarry, 1, 2-7.

Revusky, S., and Garcia, J. (1970). Learned associations over long delays. In Psychology of Learning and Motivation Advances in Research and Theory. 4, 1-84. Edited by G. H. Bower, Academic Press: New York.

Roth, M., and Kerr, T. A. (1970). Diagnosis of the reactive depressive illnesses. In Modern Trends in Psychological Medicine. 2, pp. 165-199. Edited by J. H. Price. Butterworths: London.

Rozin, P., and Kalat, J. W. (1971). Specific hungers and poison avoidance as adaptive specializations of learning. Psychological Review, 78, 459-486.

Scott, J. P. (1967). Comparative psychology and ethology. Annual Review of Psychology, 18, 65-86.

Seligman, M. E. P. (1970). On the generality of the laws of learning. Psychological Review', 77, 406-418.

Seligman, M. E. P., Maier, S. F., and Solomon, R. L. (1971). Unpredictable and uncontrollable aversive events. In Aversive Conditioning and Learning, pp. 347-400. Edited by F. R. Brush. Academic Press: New York.

Siegel, R. K. (1973). An ethological search for self-administration of hallucinogens. International Journal of the Addictions, 8, 373-393.

Snyder, S. H. (1972). Catecholamines in the brain as mediators of amphetamine psychosis. Archives of General Psychiatry, 27, 169-179.

Snyder, S. H. (1973). Amphetamine psychosis: a model schizophrenia mediated by catecholamines. American Journal of Psychiatry, 130, 61-67.

Stein, L., and Wise, C. D. (1971). Possible etiology of schizophrenia: progressive damage to the noradrenergic reward system by 6-hydroxydopamine. Science, 171, 1032-1036.

Stein, L., Wise, C. D., and Berger, B. D. (1972). Noradrenergic reward mechanisms, recovery of function, and schizophrenia. In The Chemistry of Mood, Motivation, and Memory, pp. 81-103. Edited by J. L. McGaugh, Plenum Press: New York.

Steinberg, H. (1964). Drugs and animal behaviour. British Medical Bulletin, 20, 75-80.

Tinbergen, N. (1968). On war and peace in animals and man. Science, 160, 1411-1418.

Ungerstedt, U. (1971a). Striatal dopamine release after amphetamine or nerve degeneration revealed by rotational behaviour. Acta Physiologica Scandinavica, Suppl. 367, 49-68.

Ungerstedt, U. (1971b). Postsynaptic supersensitivity after 6-hydroxy-dopamine induced degeneration of the nigrostriatal dopamine system. Acta Physiologica Scandinavica, Suppl. 367, 69-93.

Van Rossum, J. M. (1966). The significance of dopamine receptor blockade for the mechanism of action of neuroleptic drugs. Archives Internationales de Pharmacodynamie et de Thérapie, 160, 492-494. 